\title{
Emerging roles of chicken and viral microRNAs in avian disease
}

\author{
Joan Burnside*, Robin Morgan \\ From International Symposium on Animal Genomics for Animal Health (AGAH 2010) \\ Paris, France. 31 May - 2 June 2010
}

\begin{abstract}
Background: MicroRNAs are short RNAs ( $22 \mathrm{nt})$ expressed by plants, animals and viruses that regulate gene expression post-transcriptionally, and their importance is highlighted by distinct patterns of expression in many physiological processes, including development, hematopoeisis, stress resistance, and disease. Our group has characterized the microRNAs encoded by the avian herpesviruses; namely, oncogenic Marek's disease (MD) virus (MDV1), non-oncogenic MDV (MDV2) herpesvirus of turkeys (HVT), and infectious laryngotracheitis virus (ILTV).
\end{abstract}

Methods: MicroRNAs encoded by the avian herpesviruses were identified using next generation sequencing technologies (454, Illumina).

Results: The microRNAs of each the avian herpesviruses have unique sequences, but the genomic locations are similar, in that the microRNAs tend to be clustered in the rapidly evolving repeat regions of the viral genomes. For a given viral species the microRNA sequence is highly conserved in different strains with the exception of a virulence-associated polymorphism in the putative promoter of the MDV1 microRNAs upstream of the mea oncogene. These microRNAs are relatively highly expressed in tumors produced by very virulent MDV1 isolates compared to tumors produced by less virulent strains. MDV1 and HVT encode homologs of the host microRNA, miR-221, which targets a gene important in cell cycle regulation. MDV1 encodes a microRNA (mdv1-miR-M4) that shares a seed sequence with miR-155, a microRNA important in immune function. Mdv-miR-M4 is highly expressed in MDV induced tumors, while miR-155 is present at very low levels.

Conclusions: MicroRNAs are highly conserved among different field strains of MDV1, and they are expressed in lytic and latent infections and in MDV1-derived tumors. This suggests that these small molecules are very important to the virus, and roles in immune evasion, anti-apoptosis, or proliferation are likely.

\section{Background}

Marek's disease (MD) is a lymphoproliferative disorder in which aggressive T-cell lymphomas develop within two to six weeks following infection of susceptible chickens with oncogenic MD virus (MDV1) [1]. The molecular details of MDV1-induced transformation are not well understood, but vaccines developed from related, nononcogenic strains, such as MDV2 and herpesvirus of turkeys (HVT), protect animals from disease. Thus, MD is unique among virally induced lymphomas

\footnotetext{
*Correspondence: joan@udel.edu

Delaware Biotechnology Institute, University of Delaware, 15 Innovation Way, Newark, Delaware 19711, USA
}

in that highly effective vaccines exist. Despite vaccination, MD continues to be a problem worldwide, and new viruses with enhanced virulence have emerged over the last three decades. This is explained, in part, because vaccines prevent disease but not infection, and vaccinated chickens shed virulent virus into the environment. MDV1 is immunosuppressive, and one explanation for enhanced virulence of recently emerged strains is that the virus has evolved to become increasingly competent at immune system disarmament or evasion.

MDV1 is an $\alpha$-herpesvirus, and infection includes both lytic and latent stages. Productive infection is characterized by the replication of viral DNA, the synthesis of viral proteins, and ultimately the assembly of

\section{Biomed Central}


infectious virus. In the natural host, fully enveloped, infectious virus is produced only from feather follicle epithelium. A restrictive productive infection occurs in $B$ cells and chicken embryo fibroblasts (CEF) in culture, and is characterized by viral replication and viral antigen synthesis but no fully infectious virus particle production. MDV1 assumes a latent posture in T lymphocytes, with little expression of viral antigens. T lymphocytes can be transformed by MDV1, and the virus in transformed cells is generally considered to be latent, but several viral genes are transcribed in tumors as well as in lymphoblastoid cell lines derived from them. This includes meq, the candidate oncogene for MDV1 [2], as well as the latency associated transcripts (LATs) [3], which map antisense to the major transcriptional activator ICP4 and do not encode a protein product. MDV2 is a related, non-oncogenic virus that infects chickens. HVT is apathogenic and primarily infects turkeys, but has been used in combination with MDV2 as a very effective vaccine against MDV1. ILTV is more distantly related to MDV and HVT, and causes a respiratory illness in chickens.

microRNAs. MicroRNAs are short RNAs ( 22 nt) that are important post-transcriptional regulators encoded by the genomes of animals and plants [4]. Over 940 microRNAs have been discovered in the human genome [5], and genes encoding microRNAs have distinct expression profiles during development, in specific tissues, in disease, and in response to stimuli (for a recent review, see [6]). MicroRNAs are transcribed by RNA polymerase II into long primary microRNA (pri-microRNA) transcripts, which are capped and polyadenylated and can fold into characteristic hairpin structures. The primicroRNA, which can encode either a single microRNA or a cluster of several, is processed by a ribonuclease III-like enzyme, Drosha, resulting in the liberation of a 60- to 70-nucleotide RNA hairpin (pre-microRNA). The pre-microRNA is exported to the cytoplasm and further processed into a short dsRNA molecule by a second ribonuclease III-like enzyme, Dicer. A single strand of this short dsRNA, the mature microRNA, is incorporated into the RNA-induced silencing complex (RISC), which includes the endonuclease Argonaute, while the other strand, known as the star or passenger strand, is usually degraded. The RISC complex directs transcript cleavage or translational repression of target genes, and it is generally thought that the choice of mechanism depends on the degree of complementarity between the microRNA and its target site on an mRNA. In plants, microRNAs show high complementarity to their target mRNAs, and this results in endonucleolytic transcript cleavage known as 'slicing' [7]. In animals, complementarity between nucleotides $2-7 / 8$ of the microRNA (known as the 'seed' sequence) and the 3'UTR of the target mRNA appears to be crucial for directing translational repression, the predominant mechanism for microRNA action in animals (reviewed in $[8]$ ).

Herpesviruses also encode microRNAs. Herpesviruses have developed strategies to evade the host immune response during both lytic replication and persistent latent infections. Viral protein coding genes carry out varied functions important to viral survival such as blocking interferon responses, inhibiting apoptosis and interfering with innate and adaptive immune responses. The microRNAs encoded by herpesviruses are now receiving attention as critical regulators of both viral and host genes and are like to play key roles in viral survival [9].

We postulated that MDV1 and other avian herpesviruses (MDV2, HVT and infectious laryngotracheitis virus (ILTV) encode microRNAs; this paper reviews current information about avian herpesvirus microRNAs and their roles.

\section{Methods}

\section{Deep sequencing and sequence analysis}

Total RNA was purified using Trizol (Invitrogen, Carlsbad, CA), and small RNAs were purified, and cloned, and submitted to 454 (Branford, CT) or to Illumina (Hayward, CA) for sequencing. Sequence reads were compared to the chicken and viral genomes, allowing only exact matches using an in-house developed analysis pipeline. After filtering out other known small RNAs, candidate microRNA sequences mapping to unique loci were tallied to determine sequencing read frequency.

\section{Cell cultures and viruses}

CEF were prepared as described previously $[10,11]$. MDV1, MDV2 and HVT strains were from the University of Delaware collection, ILTV USDA was from ATCC via the University of Delaware collection.

\section{RNA preparation and northern blotting}

RNA was purified using Trizol, electrophoresed on acrylamide gels, and hybridized to ${ }^{32} \mathrm{P}$-labeled antisense oligonucleotide probes as described previously [12].

\section{Results and discussion}

\section{MDV1, MDV2, HVT, and ILTV encode microRNAs}

Our laboratory has identified microRNAs encoded by four avian herpesviruses, MDV1, MDV2, HVT, and ILTV $[13,14]$. Like other herpesviruses, the genome of the avian herpesviruses contains unique long $\left(\mathrm{U}_{\mathrm{L}}\right)$ and unique short $\left(\mathrm{U}_{\mathrm{S}}\right)$ regions that are flanked by terminal and internal repeats $\left(\mathrm{I} / \mathrm{TR}_{\mathrm{L}} ; \mathrm{I} / \mathrm{TR} \mathrm{S}\right)$. The unique regions contain functionally conserved protein coding sequences, while the repeats generally encode virus-specific genes. The avian herpesvirus microRNAs tend to 
be clustered in these highly plastic, repeat regions (Figure 1). In accordance with these being virus-specific regions, there is no conservation of sequence among any of the microRNAs from the different viruses. We have speculated that the viral microRNAs appeared with the evolution of the repeat regions, suggesting that the function of the microRNAs is to provide an advantage to the virus, and if their acquisition produced a loss-of-function, this could be complemented by sequences in the other repeat. Gain-of-function mutations, however, would be maintained and eventually duplicated.

In MDV1, microRNAs either flank the meq oncogene or lie in the region encoding the LATs $[13,15]$. The predominant ILTV microRNAs are antisense to the ICP4 gene, which encodes an essential immediate early transcriptional activator, and so ILTV microRNAs could serve to regulate ICP4 mRNA levels [16]. It is interesting that several of the HVT microRNAs appear to have arisen from local duplications of portions of the viral genome [14]. A striking sequence similarity between two MDV1 microRNAs (mdv1-miR-M7, M10) suggests that they also may have been generated by local duplication within the genome.

\section{Viral microRNAs are highly conserved among strains}

In contrast to the lack of sequence conservation among the different avian herpesviruses, the microRNAs of the different viral strains are highly conserved.

In MDV1, the sequence of all microRNAs was conserved among 23 different strains representing three pathotypes. However, one polymorphism in the putative promoter for the meq microRNA cluster correlates with pathogenicity, and could be responsible for the difference in expression of the meq microRNAs in tumors from very virulent pathotypes [17] (Table 1).

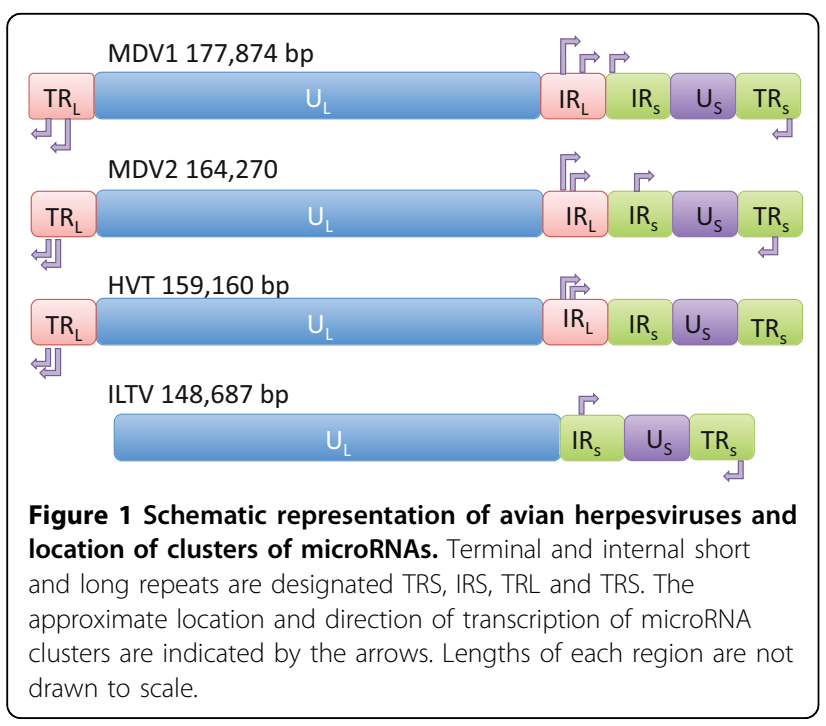

Table 1 Relative expression levels of MDV1 microRNAs in tumors produced by a very virulent + strain $(v v+, 615 K)$ compared to a very virulent strain (vv, RB1B) strain

\begin{tabular}{lc}
\hline microRNA & $\mathbf{v v}^{+} / \mathbf{v v}$ \\
\hline Upstream of meq & 3.9 \\
mdv1-miR-M2-5p & 6.4 \\
mdv1-miR-M2-3p & 1.6 \\
mdv1-miR-M3-5p & 3.3 \\
mdv1-miR-M4-5p & 3.2 \\
mdv1-miR-M5-3p & 2.3 \\
mdv1-miR-M12-3p & \\
\hline LAT region & 0.9 \\
\hline mdv1-miR-M6-5p & 1 \\
mdv1-miR-M6-3p & 1.1 \\
mdv1-miR-M7-5p & 0.7 \\
mdv1-miR-M7-3p & 0.9 \\
mdv1-miR-M8-5p & 0.9 \\
mdv1-miR-M8-3p &
\end{tabular}

Ratios were derived from average densitometry of northern blot analysis of samples from two different chickens for each strain. Expression of viral DNA was comparable in all samples. U6 was used as a loading control and showed a ratio of 0.8 .

Only two strains of HVT (FC126, PB1) and two MDV2 strains (SB1 and HPRS-24) are available for sequence comparison. In preliminary studies, no polymorphisms have been identified in microRNAs of MDV2, while three polymorphisms were found in HVT PB1 microRNAs (hvt-miR-H3-5p;12-5p;16-5p) compared to the reference strain FC126. In both hvt-miRH12-5p and 16-5p, the polymorphism is located within the seed sequence. Similarly, the sequence of the predominant ILTV microRNAs, iltv-miR-I5 and I6, are highly conserved; these microRNAs lie antisense to the ICP4 gene and this region is identical in the 22 strains of ILTV included in GenBank.

Overall, the high level of conservation supports the idea that there is selective pressure to maintain the microRNA sequences, inferring that viral microRNAs have important functions [18]. Despite the lack of sequence identity among the microRNAs of the different avian herpesviruses, there may be overlaps in function. Typically, a microRNA binds to the 3'UTR of target genes, with binding mediated through the 'seed' sequence (nt 2-7/8) of the microRNA [19]. Thus, the same gene could be targeted at different sequences by different microRNAs, and some of these divergent avian herpesviral microRNAs could share targets.

\section{Viral orthologs of host microRNAs}

One of the HVT microRNAs, hvt-miR-H14-3p, is a homologue of gga-miR-221 with 21/23 nucleotide identity, including complete conservation of the seed/family sequence. There is sequence similarity with downstream 
flanking region in the chicken genome as well [14]. In the chicken, miR-221 is very abundant in CEFs and other tissues [12,20,21], and it is likely that expression is similar in the turkey, which is the natural host of HVT. Thus it is possible that this HVT homologue was captured from the host genome, either through propagation in tissue culture or during infection and replication in the host. MDV1 contains a microRNA (mdv1-miR-M32) that shares a seed sequence with miR-221 \{Morgan, 2008 \#4818\}. miR-221 targets the cyclin-dependent kinase inhibitor 1B (p27, Kip1), a regulator of the cell cycle $\mathrm{G} 1$ to $\mathrm{S}$ phase transition. The repression of $\mathrm{p} 27$ by miR-221 is thought to play an important role in cancer progression [22,23], and could play a role in MDV1induced tumorigenesis \{Lambeth, 2009 \#5006\}. Although HVT is non-oncogenic, expression of a miR-221 ortholog and down-regulation of p27 could move the cell cycle to the $\mathrm{S}$ phase in order to support replication of the viral genome as well as to increase growth of infected cells for additional viral production.

MDV1, like Kaposi's sarcoma herpesvirus (KSHV), encodes a microRNA that shares a seed sequence with the host microRNA, miR-155 (kshv-miR-K11; mdv1miR-M4). It has been proposed that kshv-miR-K11 is a functional ortholog of mir-155 and can regulate the same set of host mRNAs, and this contributes to KSHV-induced tumorigenesis [24], [25]. Because the host response to infection must be balanced between elimination of pathogens and limited damage to host cells, microRNAs are ideal candidates for fine-tuning of the rapidly changing immune response. However, the timing and cellular location of expression are critical for manifesting function of immunomodulatory microRNAs. For miR-155, loss- and gain-of-function studies have demonstrated a critical role in both immune cell development and function. Sustained expression of miR-155 can stimulate production of granulocytes, and miR-155 over-expression can lead to neoplasia [26]. However, miR-155 expression is complicated in that its induction in macrophages by activation of the immune response with lipopolysaccharides does not result in subsequent neoplasia [27,28]. Mdv1-miR-M4 also shares some targets with mir-155, and it has been suggested that mdv1miR-M4 target regulation plays a role in MDV-induced lymphomagenesis [29]. In deep sequencing studies of MDV-induced splenic tumors, we have noted decreased levels of miR-155 (and most microRNAs) compared to normal spleen, resting $\mathrm{T}$ cells, or activated $\mathrm{T}$ cells (Table 2). An overall decline in microRNA expression has been noted in other cancers [30,31], and we hypothesize that mdv1-miR-M4 serves to replace miR155 in regulating functions essential to the phenotype of the transformed cell. In addition, as noted above, tumors produced by more aggressive strains of MDV1 express
Table 2 Sequencing frequencies of immune-related (A) and MDV (B) microRNAs in spleen, $T$ cells, activated $T$ cells (a-T), and MDV-induced splenic tumors

\begin{tabular}{|c|c|c|c|c|}
\hline & spleen & T cells & a- $T$ cells & tumor \\
\hline \multicolumn{5}{|l|}{$\bar{A}$} \\
\hline gga-miR-21 & 15779 & 4441 & 3002 & 693 \\
\hline gga-miR-181a & 2626 & 1507 & 11602 & 54 \\
\hline gga-miR-181b & 727 & 956 & 9458 & 59 \\
\hline gga-miR-221 & 2326 & 2679 & 13295 & 83 \\
\hline gga-miR-222 & 324 & 1166 & 21646 & 72 \\
\hline gga-miR-146a & 794 & 407 & 1165 & 36 \\
\hline gga-miR-146b & 2154 & 1016 & 11865 & 857 \\
\hline gga-miR-155 & 499 & 1403 & 52081 & 29 \\
\hline gga-miR-92 & 973 & 1943 & 1799 & 211 \\
\hline \multicolumn{5}{|l|}{ B } \\
\hline$m d v-m i R-M 2-5 p$ & & & & 104 \\
\hline mdv-miR-M2-3p & & & & 591 \\
\hline$m d v-m i R-M 3-5 p$ & & & & 187 \\
\hline mdv-miR-M3-3p & & & & 50 \\
\hline mdv-miR-M4 & & & & 6682 \\
\hline mdv-miR-M5 & & & & 581 \\
\hline$m d v-m i R-M 8-5 p$ & & & & 345 \\
\hline mdv-miR-M9-5p & & & & 63 \\
\hline mdv-miR-M9-3p & & & & 74 \\
\hline mdv-miR-M12-3p & & & & 164 \\
\hline$m d v-m i R-M 10-5 p$ & & & & 12 \\
\hline
\end{tabular}

higher levels of mdv1-miR-M4 and other microRNAs in this cluster (Table 1), consistent with a role in tumorigenesis. To further elucidate the role of mdv1-miR-M4, our laboratory has constructed arecombinant HVT that expresses mdv-miR-M4. The recombinant virus showed improved growth characteristics in vitro and in vivo implying that this microRNA can confer replicative advantages to the virus, but mdv1-miR-M4 is not turmorigenic in the context of HVT (unpublished results). Thus the MDV1 ortholog of miR-155 may also be pleomorphic in that it facilitates viral growth by impeding the apoptotic response to infection and also down-regulates genes involved in cell cycle control.

\section{Conclusions}

The presence of microRNAs in species-specific repeat regions of the genomes of avian herpesvirus and the conservation among strains in a particular virus all point to the importance of these gene regulators in virus survival. While the functions of the viral microRNAs have not been established, it is apparent that these are ideally designed for fine-tuning the host response to infection with viruses that replicate and also form persistent latent infections in the host. Maintaining cell viability and establishing a permissive environment for unregulated synthesis of foreign DNA requires 
controlling the cell cycle, blocking apoptosis and escaping the innate immune response to infection; genes regulating these pathways are among the potential targets for these molecules. In addition, microRNAs are emerging as therpeutic targets, and understanding their roles in viral infection has great potential for the development of improved treatments and vaccines.

\section{Acknowledgements}

We value the contributions of our coworkers (Amy Anderson, Erin Bernberg, Lisa Waidner, Milos Markis and Grace Lagasse) and collaborators (Pam Green and Blake Meyers) in these studies. This work was supported by USDA grants.

This article has been published as part of BMC Proceedings Volume 5 Supplement 4, 2011: Proceedings of the International Symposium on Animal Genomics for Animal Health (AGAH 2010). The full contents of the supplement are available online at http://www.biomedcentral.com/1753$6561 / 5$ ? issue $=\$ 4$.

\section{Authors' contributions}

$J B$ and RM directed the research and wrote the manuscript.

\section{Competing interests}

The authors declare that they have no competing interests.

Published: 3 June 2011

\section{References}

1. Witter RL, Schat K: Marek's Disease. In Diseases of Poultry.. 11 edition. Ames, lowa: lowa State Press:Saif YM 2003:407-464

2. Kung H, Xia L, Brunovskis P, Li D, Liu J, Lee L: Meq: an MDV-specific bZIP transactivator with transforming properties. Curr Top Microbiol Immunol 2001, 255:245-260

3. Morgan RW, Xie Q, Cantello JL, Miles A, Bernberg E, Kent J, Anderson AS: Marek's disease virus latency. In Marek's Disease. Volume 255. New York: Springer; Hirai K 2001:223-243.

4. Lagos-Quintana M, Rauhut R, Lendeckel W, Tuschl T: Identification of novel genes coding for small expressed RNAs. Science 2001, 294:853-858.

5. Griffiths-Jones S, S HK, Dongen Sv, Enright AJ: miRBase: tools for microRNA genomics. Nucleic Acids Research 2008, 36:D154-D158.

6. Stefani G, Slack FJ: Small non-coding RNAs in animal development. Nat Rev Mol Cell Biol 2008, 9:219-230.

7. Rhoades MW, Reinhart BJ, Lim LP, Burge CB, Bartel B, Bartel DP: Prediction of plant microRNA targets. Cell 2002, 110:513-520.

8. Filipowicz W, Bhattacharyya SN, Sonenberg N: Mechanisms of posttranscriptional regulation by microRNAs: Are the answers in sight? Nature Reviews: Genetics 2008, 9:102-114.

9. Sullivan CS, Cullen BR: Non-coding regulatory RNAs of the DNA tumor viruses. In DNA Tumor Viruses. New York: Springer;Damania B, Pipas JM 2009:

10. Schat KA, Purchase HG: Isolation and Identification of Avian Pathogens, American Association of Avian Pathologists. Kennett Square, Pennsylvania, USA; 1998, 223-234.

11. Tannock GA, Bryce DA, Paul JA: Evaluation of chicken kidney and chicken embryo kidney cultures for the large-scale growth of attenuated influenza virus master strain A/Ann/Arbor/6/60-ca. Vaccine 1985, 3(3):333-339.

12. Burnside J, Bernberg E, Anderson A, Lu C, Meyers BC, Green PJ, Jain N, Isaacs GK, Morgan RW: Marek's disease virus microRNAs map to meq and LAT. J Virol 2006, 80:8778-8786.

13. Burnside J, Bernberg E, Anderson A, Lu C, Meyers BC, Green PJ, Jain N, Isaacs G, Morgan RW: Marek's disease virus microRNAs map to meq and latency associated transcript. J Virology 2006, 80:8778-8786.

14. Waidner L, Morgan R, Anderson A, Bernberg E, Kamboj S, Garcia M, Riblet S, Ouyang M, Isaacs G, Markis M, et al: Novel ILTV and HVT microRNAs have conserved genomic locations with those of other Gallid and Meleagrid herpesvirus microRNAs. Virology 2009, 338:128-136.
15. Yao Y, Zhao Y, Xu H, Smith LP, Lawrie CH, Watson M, Nair V: MicroRNA profile of Marek's disease virus-transformed T-cell line MSB-1: Predominance of virus-encoded microRNAs. J Virology 2008, 82:4007-4015.

16. Waidner LA, Morgan RW, Anderson Amy S., Bernberg EL, German MA, Meyers BC, Green PJ, Burnside J: A microRNA of Infectious Laryngotracheitis Virus downregulate the major transcription factor ICP4. Keystone Symposium 2010.

17. Morgan R, Anderson A, Bernberg E, Kamboj S, Huang E, Lagasse G, Isaacs $G$, Parcells M, Meyers BC, Green PJ, et al: Sequence conservation and differential expression of Marek's disease virus microRNAs. J Virology 2008, 82:12213-12220.

18. Lagasse G: Sequence conservation of microRNAs in Marek's disease viruses and in herpesviruses of turkeys. Thesis University of Delaware 2010.

19. Bartel DP: MicroRNAs: Genomics, biogenesis, mechanism, and function. Cell 2004, 116(2):281-297.

20. Burnside J, Morgan RW, Green PJ, Meyers BC, Nobuta K, Kamboj S, Gupta R, McCormick K, Shridhara G, Nakano M, et al: Gallus gallus sbs.[http://mpss. udel.edu/gga/].

21. Burnside J, Ouyang M, Anderson A, Bernberg E, Lu C, Meyers BC, Green PJ, Markis $M$, Isaacs GK, Huang $E$, et al: Deep sequencing of chicken microRNAs. BMC Genomics 2008, 9(185), doi:10.1186/1471-2164-1189-1185.

22. Fornari F, Gramantieri L, Ferracin M, Veronese A, Sabbioni S, Calin GA, Grazi GL, Giovannini C, Croce CM, Bolondi L, et al: MiR-221 controls CDKN1C//p57 and CDKN1B//p27 expression in human hepatocellular carcinoma. Oncogene 2008, advanced on-line publication, June 2.

23. Galardi S, Mercatelli N, Giorda E, Massalini S, Frajese GV, Ciafre SA, Farace MG: miR-221 and miR-222 Expression Affects the Proliferation Potential of Human Prostate Carcinoma Cell Lines by Targeting p27Kip1. J Biol Chem 2007, 282(32):23716-23724.

24. Gottwein E, Mukherjee N, Sachse C, Frenzel C, Majoros WH, Chi J-TA, Braich R, Manoharan M, Soutschek J, Ohler U, et al: A viral microRNA functions as an orthologue of cellular miR-155. Nature 2007, 450:1096-1099.

25. Skalsky RL, Samols MA, Plaisance KB, Boss IW, Riva A, Lopez MC, Baker HV Renne R: Kaposi's sarcoma-associated herpesvirus encodes an ortholog of miR-155. J Virology 2007, 81(23):12836-12845.

26. O'Connell RM, Rao DS, Chaudhuri AA, Boldin MP, Taganov KD, Nicoll J, Paquette RL, Baltimore D: Sustained expression of microRNA-155 in hematopoietic stem cells causes a myeloproliferative disorder. The Journal of Experimental Medicine 2008, 205(3):585-594.

27. O'Connell RM, Taganov KD, Boldin MP, Cheng G, Baltimore D: MicroRNA155 is induced during the macrophage inflammatory response. PNAS 2007, 104(5):1604-1609.

28. Taganov KD, Boldin MP, Chang K-J, Baltimore D: NF-KB-dependent induction of microRNA miR-146, an inhibitor targeted to signaling proteins of innate immune responses. PNAS 2006, 103(33):12481-12486.

29. Zhao Y, Yao Y, Xu H, Lambeth L, Smith LP, Kgosana L, Wang X, Nair V: A functional microRNA-155 ortholog encoded by the oncogenic Marek's Disease Virus. J Virology 2009, 83(1):489-492.

30. Thomson JM, Newman M, Parker JS, Morin-Kensicki EM, Wright T, Hammond SM: Extensive post-transcriptional regulation of microRNAs and its implications for cancer. Genes Dev 2006, 20(16):2202-2207.

31. Lu J, Getz G, Miska EA, Alvarez-Saavedra E, Lamb J, Peck D, SweetCordero A, Ebert BL, Mak RH, Ferrando AA, et al: MicroRNA expression profiles classify human cancers. Nature 2005, 435:834-843.

doi:10.1186/1753-6561-5-S4-S2

Cite this article as: Burnside and Morgan: Emerging roles of chicken and viral microRNAs in avian disease. BMC Proceedings 2011 5(Suppl 4):S2. 